\title{
The Explicit Algebraic Reynolds Stress Models for Turbulent Flows
}

\author{
Hoang Pham ${ }^{1} \&$ Tuyen D. Nguyen ${ }^{2}$ \\ ${ }^{1}$ University of Texas at Arlington, Arlington, USA \\ ${ }^{2}$ California State University, 800 N. State College, Fullerton, USA \\ Correspondence: Tuyen D. Nguyen, California State University, 800 N. State College, Fullerton, CA 92834, \\ USA. E-mail: trentspringfield@yahoo.com
}

Received: December 12, 2011

Accepted: January 2, 2012 Online Published: May 27, 2012

doi:10.5539/mer.v2n1p95

URL: http://dx.doi.org/10.5539/mer.v2n1p95

\begin{abstract}
The explicit algebraic Reynolds stress models are obtained from second-order closure models that are valid for three-dimensional turbulent flows in non-inertial frames. The purpose of this present research is to simplify the development of the Reynolds stress anisotropy tensor. This anisotropy stress tensor has seven scalar coefficients and has seven tensor polynomial groups that are the integrity basis for the functions of both symmetric and antisymmetric tensors. This research will also explicitly determine the six independent invariants of the mean strain rate tensor and of the mean rotation rate tensor. The resulting algebraic equation for the anisotropy tensor depends on the choice of the model that is used to determine the dissipation rate and pressure-strain correlation. These equations also represent the slow pressure strain rate and an isotropic dissipation rate tensor of the Rotta model. The results of present research can be compared with the results of Gatski and Speziale that give the complete expression for a traceless symmetric second order tensor which depended on the symmetric and the antisymmetric tensor that involved ten tensor polynomial groups with five independent invariants. The present work reduces the ten tensor polynomial groups down to seven groups which drastically decreases computational time.
\end{abstract}

Keywords: explicit algebra, Reynolds stress models, turbulent flows, engineering applications

\section{Introduction}

Turbulent flows occur naturally in nature as well as in engineering applications on many levels. For example, in nature one can observe turbulent flows that occur in the water below the surface of an ocean, in a river, in atmosphere and even in the lungs of a human being. With respect to mechanical engineering, turbulent flows occur in when one drives a car, flies an airplane, cools, and heats one's home, etc. The Navier-Stokes equation can properly describe the details of turbulent motions (Spencer \& Rivin, 1958; Spencer, 1971). The ensemble averaged Navier-Stokes equations are often sufficient and practical to describe the turbulent motions in engineering problems. In taking an average of the Navier-Stokes equations for turbulent flow, which is three-dimensional, unsteady, irregular, random, and rotational one must find the Reynolds stress equation that appears in the Navier-Stokes equation (Pope, 1975). This is accomplished by multiplying the Navier-Stokes equation by a fluctuating property and by the time average product. Using this procedure a differential equation for the Reynolds stress tensor can be derived (Daly \& Harlow, 1970).

The purpose of the present research is to obtain the Reynolds stress anisotropy tensor for the turbulent flows by determining the seven $\mathrm{L}_{\mathrm{i}}$ coefficients. These coefficients are based from the Reynolds stress transport equation (Lumley, 1970). The terms of the Reynolds stress tensor are the pressure-strain correlation $\Pi_{i j}$, the dissipation-rate correlation $\varepsilon_{i j}$, and third-order diffusion correlation $C_{i j k}$, where the pressure-strain correlation $\Pi_{i j}$; is a function related to the anisotropy tensor $\mathrm{b}_{\mathrm{ij}}$, the turbulent kinetic energy $\mathrm{K}$, the scalar turbulent dissipation rates $\varepsilon, \mathrm{A}_{\mathrm{ij}}$ and $\mathrm{M}_{\mathrm{ijk}} ; \mathrm{A}_{\mathrm{ij}}$ and $\mathrm{M}_{\mathrm{ijk}}$ are actually functions, in time and are wave-number spaces, of the energy spectrum tensor (Launder, Reece, \& Rodi, 1975). The model for the pressure-strain correlation can be written in equivalent form as a function of the anisotropy tensor $b_{i j}$, the mean strain rate tensor $S_{i j}$ and the mean rotation rate tensor $\mathrm{W}_{\mathrm{ij}}$ (Rodi, 1996).

The anisotropy tensor $b_{i j}$ is a function of the pressure-strain correlation, and the main issue in obtaining this anisotropy tensor is the determination of the seven $\mathrm{L}_{\mathrm{i}}$ coefficients, which are scalar functions, that involves seven tensor polynomial groups with six independent invariants. 


\section{Present Research}

In the present research, for three dimensional turbulent flows the anisotropy tensor $b_{i j}$ shown as follows:

$$
\mathrm{b}_{\mathrm{ij}}=\sum_{i=1}^{7} \mathrm{~L}_{\mathrm{i}} \mathrm{w}_{\mathrm{i}}
$$

where $\mathrm{w}_{\mathrm{i}}$ is the integrity basis for the functions of a symmetric and antisymmetric tensor of the mean velocity gradient and $\mathrm{L}_{\mathrm{i}}$ are scalar functions depend on the six of the irreducible invariants of $\mathrm{S}_{\mathrm{ij}}$ and $\mathrm{W}_{\mathrm{ij}}$. Let the integrity basis $\mathrm{w}_{\mathrm{i}}$ as follows:

$$
\begin{aligned}
& \mathrm{w}_{0}=\mathrm{I} \\
& \mathrm{w}_{1}=\mathrm{v}_{1} \\
& \mathrm{w}_{2}=\mathrm{v}_{2} \\
& \mathrm{w}_{3}=\mathrm{v}_{3}-\mathrm{g}_{31} \mathrm{w}_{1}-\mathrm{g}_{30} \mathrm{I} \\
& \mathrm{w}_{4}=\mathrm{v}_{4}-\mathrm{g}_{43 \mathrm{~b}} \mathrm{w}_{3}-\mathrm{g}_{41} \mathrm{w}_{1}-\mathrm{g}_{40} \mathrm{I} \\
& \mathrm{w}_{5}=\mathrm{v}_{5}-\mathrm{g}_{52} \mathrm{w}_{2} \\
& \mathrm{w}_{6}=\mathrm{v}_{6}-\mathrm{g}_{65 \mathrm{~b}} \mathrm{w}_{5}-\mathrm{g}_{64 \mathrm{~b}} \mathrm{w}_{4}-\mathrm{g}_{63 \mathrm{~b}} \mathrm{w}_{3}-\mathrm{g}_{61} \mathrm{w}_{1}-\mathrm{g}_{60} \mathrm{I} \\
& \mathrm{w}_{7}=\mathrm{v}_{7}-\mathrm{g}_{75 \mathrm{~b}} \mathrm{w}_{5}-\mathrm{g}_{74 \mathrm{~b}} \mathrm{w}_{4}-\mathrm{g}_{73 \mathrm{~b}} \mathrm{w}_{3}-\mathrm{g}_{72} \mathrm{w}_{2}
\end{aligned}
$$

and

$$
\begin{aligned}
& \mathrm{v}_{8}=\mathrm{g}_{85 \mathrm{~b}} \mathrm{~W}_{5}+\mathrm{g}_{84 \mathrm{~b}} \mathrm{~W}_{4}+\mathrm{g}_{82} \mathrm{~W}_{2} \\
& \mathrm{v}_{9}=\mathrm{g}_{96 \mathrm{~b}} \mathrm{w}_{6}+\mathrm{g}_{95 \mathrm{~b}} \mathrm{w}_{5}+\mathrm{g}_{94 \mathrm{~b}} \mathrm{w}_{4}+\mathrm{g}_{93 \mathrm{~b} \mathrm{w}_{3}}+\mathrm{g}_{92} \mathrm{w}_{2}+\mathrm{g}_{91} \mathrm{w}_{1}+\mathrm{g}_{90} \mathrm{I} \\
& \mathrm{v}_{10}=\mathrm{g}_{107 \mathrm{~b}} \mathrm{~W}_{7}+\mathrm{g}_{105 \mathrm{~b}} \mathrm{~W}_{5}+\mathrm{g}_{104 \mathrm{~b}} \mathrm{~W}_{4}+\mathrm{g}_{103 \mathrm{~b}} \mathrm{~W}_{3}+\mathrm{g}_{102} \mathrm{w}_{2}+\mathrm{g}_{101} \mathrm{w}_{1}
\end{aligned}
$$

where the mean strain rate tensor $S_{i j}$ and the mean rotation rate tensor $W_{i j}$ are the symmetric and antisymmetric parts of the mean velocity gradient tensor as follows :

$$
\begin{gathered}
\mathrm{S}_{\mathrm{ij}}=\frac{1}{2}\left(\frac{\partial U_{i}}{\partial x_{j}}+\frac{\partial U_{j}}{\partial x_{i}}\right) \quad \mathrm{W}_{\mathrm{ij}}=\frac{1}{2}\left(\frac{\partial U_{i}}{\partial x_{j}}-\frac{\partial U_{j}}{\partial x_{i}}\right) \\
\mathrm{g}_{107 \mathrm{~b}}=\frac{\operatorname{trace}\left(v_{10} \cdot w_{7}\right)}{\operatorname{trace}\left(w_{7} \cdot w_{7}\right)} \text { and } \mathrm{g}_{31}=\frac{\operatorname{trace}\left(v_{3} \cdot v_{1}\right)}{\operatorname{trace}\left(v_{1} \cdot v_{1}\right)}
\end{gathered}
$$

I denotes the unit tensor and let $\mathrm{v}_{\mathrm{i}}$ is the functions of a symmetric and antisymmetric tensor as follows:

$$
\begin{aligned}
& \mathrm{v}_{1}=\mathrm{S} \\
& \mathrm{v}_{2}=\mathrm{S} \mathrm{W}-\mathrm{W} \mathrm{S} \\
& \mathrm{v}_{3}=\mathrm{S}^{2} \\
& \mathrm{v}_{4}=\mathrm{W}^{2} \\
& \mathrm{v}_{5}=\mathrm{W} \mathrm{S}^{2}-\mathrm{S}^{2} \mathrm{~W} \\
& \mathrm{v}_{6}=\mathrm{S} \mathrm{W}^{2}+\mathrm{W}^{2} \mathrm{~S} \\
& \mathrm{v}_{7}=\mathrm{W} \mathrm{S} \mathrm{W}-\mathrm{W}^{2} \mathrm{~S} \mathrm{~W} \\
& \mathrm{v}_{8}=\mathrm{SW} \mathrm{S}^{2}-\mathrm{S}^{2} \mathrm{~W} \mathrm{~S} \\
& \mathrm{v}_{9}=\mathrm{W}^{2} \mathrm{~S}^{2}+\mathrm{S}^{2} \mathrm{~W}^{2} \\
& \mathrm{v}_{10}=\mathrm{W} \mathrm{S}^{2} \mathrm{~W}^{2}-\mathrm{W}^{2} \mathrm{~S}^{2} \mathrm{~W}
\end{aligned}
$$

and let the six irreducible invariants of $\mathrm{S}_{\mathrm{ij}}$ and $\mathrm{W}_{\mathrm{ij}}$ are:

$$
\begin{aligned}
& \mathrm{I}_{1}=<\mathrm{S}^{2}> \\
& \mathrm{I}_{2}=<\mathrm{W}^{2}> \\
& \mathrm{I}_{3}=<\mathrm{S}^{3}> \\
& \mathrm{I}_{4}=<\mathrm{SW}^{2}> \\
& \mathrm{I}_{5}=<\mathrm{S}^{2} \mathrm{~W}^{2}>
\end{aligned}
$$




$$
\mathrm{I}_{6}=<\mathrm{S}^{2} \mathrm{~W}^{2} \mathrm{~S} \mathrm{~W}>
$$

In the present research, an explicit relationship for the anisotropy tensor is computed by two methods. The coefficients $L_{i}$ are determined by using two methods then the numerical results of the anisotropy tensor $b_{i j}$ of the present research are compared with the numerical results of Gatski and Speziale (1993), from these results it will be clear that all three methods will have the exact same numerical results. Therefore the anisotropy tensor can then be written as follows:

\section{Calculation and Numerical Results}

$$
\mathrm{b}_{\mathrm{ij}}=\sum_{i=1}^{10} G_{i} T_{i}=\sum_{i=1}^{7} L_{i} w_{i}
$$

\section{Method One: Calculation of the Anisotropy Tensor}

To find the explicit expression for the anisotropy tensor that requires some knowledge of basic in linear algebra, the theory of invariants and the theory of matrix polynomials and its application to fluid mechanics. To determine $\mathrm{L}_{\mathrm{i}}$ are the scalar functions of the six irreducible invariants of $\mathrm{S}_{\mathrm{ij}}$ and $\mathrm{W}_{\mathrm{ij}}$. First the matrix $\mathrm{H}_{\mathrm{ij}}$, that is [7x7] matrix must be determined.

$$
\sum_{i=1}^{7} \delta_{1 i}=\sum_{i=1, j=1}^{7}\left(-\delta_{i j}-\mathrm{H}_{\mathrm{ij}}+\mathrm{J}_{\mathrm{ij}}\right) \mathrm{L}_{\mathrm{j}}
$$

Where

$$
\mathrm{A}_{\mathrm{ji}}=-\delta_{\mathrm{ij}}-\mathrm{H}_{\mathrm{ij}}+\mathrm{J}_{\mathrm{ij}}
$$

and let

$$
\begin{aligned}
& \mathrm{B}_{\mathrm{i}}=\sum_{i=1}^{7} \delta_{1 i} \\
& \mathrm{~B}_{\mathrm{i}}=\mathrm{A}_{\mathrm{ij}} \mathrm{L}_{\mathrm{j}}
\end{aligned}
$$

Thus

$$
\mathrm{L}_{\mathrm{j}}=A_{j 1}{ }^{-1}
$$

where $\mathrm{L}_{\mathrm{i}}, \mathrm{i}=1,2,3,4,5,6$, and 7 are the solutions of the explicit expression for the anisotropy tensor. The present numerical results can be compared with the numerical results of Gatski and Speziale, it can be seen that both of them have exactly the same numerical results for the anisotropy tensor $b_{i j}$, that is:

$$
\mathrm{b}_{\mathrm{ij}}=\sum_{i=1}^{10} G_{i} T_{i}=\sum_{i=1}^{7} L_{i} w_{i}
$$

The solutions of the matrices $\mathrm{A}_{\mathrm{ji}}, \mathrm{H}_{\mathrm{ij}}$ and $\mathrm{J}_{\mathrm{ij}}$.

1.

$$
\mathrm{w}_{1} \mathrm{~S}+\mathrm{S} \mathrm{w}_{1}-2 / 3<\mathrm{w}_{1} \mathrm{~S}>\mathrm{I}=\mathrm{S} \mathrm{S}+\mathrm{S} \mathrm{S}-2 / 3<\mathrm{S} \mathrm{S}>\mathrm{I}=2 \mathrm{~S}^{2}-2 / 3 \mathrm{I}_{1} \mathrm{I}
$$

Where

$$
\mathrm{S}^{2}=\mathrm{v}_{3}=\mathrm{w}_{3}+\mathrm{g}_{31} \mathrm{v}_{1}+\mathrm{g}_{30} \mathrm{I},<\mathrm{S}^{2}>=\mathrm{I}_{1}
$$

and

$$
\mathrm{g}_{30}=\frac{\operatorname{trace}\left(v_{3} . I\right)}{\operatorname{trace}(I . I)}=\frac{\operatorname{trace}\left(S^{2} \cdot I\right)}{3}=\frac{I_{1}}{3}
$$

then

$$
2\left(S^{2}-1 / 3 I_{1} I\right)=2\left(w_{3}+g_{31} v_{1}+g_{30} I-1 / 3 I_{1} I\right)
$$

Thus,

$$
\mathrm{w}_{1} \mathrm{~S}+\mathrm{S} \mathrm{w}_{1}-2 / 3<\mathrm{w}_{1} \mathrm{~S}>\mathrm{I}=2 \mathrm{w}_{3}+2 \mathrm{~g}_{31} \mathrm{w}_{1}
$$

2. $\mathrm{W}_{2} \mathrm{~S}+\mathrm{S} \mathrm{W}_{2}-2 / 3<\mathrm{W}_{2} \mathrm{~S}>\mathrm{I}=(\mathrm{S} \mathrm{W}-\mathrm{W} \mathrm{S}) \mathrm{S}+\mathrm{S}(\mathrm{S} \mathrm{W}-\mathrm{W} \mathrm{S})-2 / 3<(\mathrm{S}$ W $-\mathrm{W} \mathrm{S}) \mathrm{S}>\mathrm{I}$ 


$$
=\mathrm{SW} \mathrm{S}-\mathrm{W} \mathrm{S}^{2}+\mathrm{S}^{2} \mathrm{~W}-\mathrm{S} \mathrm{W} \mathrm{S}-2 / 3<\mathrm{SW} \mathrm{S}-\mathrm{W} \mathrm{S}^{2}>\mathrm{I}
$$

then

$$
=-\mathrm{W} \mathrm{S}+\mathrm{S}^{2} \mathrm{~W}=-\mathrm{v}_{5}=-\mathrm{W}_{5}-\mathrm{g}_{52} \mathrm{~W}_{2}
$$

Thus,

$$
\mathrm{w}_{2} \mathrm{~S}+\mathrm{S} \mathrm{w}_{2}-2 / 3<\mathrm{w}_{2} \mathrm{~S}>\mathrm{I}=-\mathrm{w}_{5}-\mathrm{g}_{52} \mathrm{w}_{2}
$$

3. $\mathrm{w}_{3} \mathrm{~S}+\mathrm{S} \mathrm{w}_{3}-2 / 3<\mathrm{w}_{3} \mathrm{~S}>\mathrm{I}=\left(\mathrm{v}_{3}-\mathrm{g}_{31} \mathrm{v}_{1}-\mathrm{g}_{30} \mathrm{I}\right) \mathrm{S}+\mathrm{S}\left(\mathrm{v}_{3}-\mathrm{g}_{31} \mathrm{v}_{1}-\mathrm{g}_{30} \mathrm{I}\right)-2 / 3<\left(\mathrm{v}_{3}-\mathrm{g}_{31} \mathrm{v}_{1}-\mathrm{g}_{30} \mathrm{I}\right) \mathrm{S}>\mathrm{I}$

$$
\begin{aligned}
& =S^{3}-g_{31} S^{2}-g_{30} S+S^{3}-g_{31} S^{2}-g_{30} S-2 / 3<S^{3}-g_{31} S^{2}> \\
& =2 S^{3}-2 g_{31} S^{2}-2 g_{30} S-2 / 3 I_{3}+2 / 3\left(I_{3} / I_{1}\right) I_{1}
\end{aligned}
$$

where $<\mathrm{g}_{30} \mathrm{~S}>=0, \mathrm{~S}=\mathrm{v}_{1}=\mathrm{w}_{1}$ and $\mathrm{g}_{31}=\mathrm{I}_{3} / \mathrm{I}_{1}$

From 3.8, let $\mathrm{a}=\mathrm{S}$ then

$$
\mathrm{S}^{3}=-1 / 2 \mathrm{~S}\left(-<\mathrm{S}^{2}>\right)+\mathrm{I} \operatorname{det} \mathrm{S},
$$

Where

$$
\operatorname{det} \mathrm{S}=1 / 3<\mathrm{S}^{3}>=\mathrm{I}_{3} / 3
$$

then

$$
\mathrm{S}^{3}=\mathrm{I}_{1} / 2 \mathrm{w}_{1}+\mathrm{I}_{3} / 3
$$

and

$$
\mathrm{S}^{2}=\mathrm{v}_{3}=\mathrm{w}_{3}+\mathrm{g}_{31} \mathrm{w}_{1}+\mathrm{g}_{30} \mathrm{I}
$$

then equation (3.8) becomes as

$$
=\mathrm{I}_{1} \mathrm{w}_{1}+2 / 3 \mathrm{I}_{3}-2 \mathrm{~g}_{31}\left(\mathrm{w}_{3}+\mathrm{g}_{31} \mathrm{w}_{1}+\mathrm{g}_{30} \mathrm{I}\right)-2 \mathrm{~g}_{30} \mathrm{w}_{1}
$$

where

$$
-2 g_{31} g_{30}=-2 / 3 I_{3}
$$

Thus,

Similarly

4.

$$
\mathrm{w}_{3} \mathrm{~S}+\mathrm{S} \mathrm{w}_{3}-2 / 3<\mathrm{w}_{3} \mathrm{~S}>\mathrm{I}=\mathrm{w}_{1}\left(\mathrm{I}_{1}-2 \mathrm{~g}_{31}^{2}-2 \mathrm{~g}_{30}\right)-2 \mathrm{~g}_{31} \mathrm{w}_{3}
$$

4.

5.

$$
\begin{aligned}
& \mathrm{w}_{4} \mathrm{~S}+\mathrm{S} \mathrm{w}_{4}-2 / 3<\mathrm{w}_{4} \mathrm{~S}>\mathrm{I}=\mathrm{w}_{1}\left[\mathrm{~g}_{61}-\mathrm{I}_{1} \mathrm{~g}_{43 \mathrm{~b}}+2\left(\mathrm{~g}_{43 \mathrm{~b}} \mathrm{~g}_{31}{ }^{2}-\mathrm{g}_{41} \mathrm{~g}_{31}\right)+\right. \\
& \left.2\left(\mathrm{~g}_{43 \mathrm{~b}} \mathrm{~g}_{30}-\mathrm{g}_{40}\right)\right]+\mathrm{w}_{3}\left[\mathrm{~g}_{63 \mathrm{~b}}+2\left(\mathrm{~g}_{43 \mathrm{~b}} \mathrm{~g}_{31}-\mathrm{g}_{41}\right)\right]+\mathrm{g}_{64 b} \mathrm{w}_{4}+\mathrm{g}_{65 \mathrm{~b}} \mathrm{w}_{5}+\mathrm{w}_{6}
\end{aligned}
$$

$$
\mathrm{w}_{5} \mathrm{~S}+\mathrm{S} \mathrm{w}_{5}-2 / 3<\mathrm{w}_{5} \mathrm{~S}>\mathrm{I}=\mathrm{w}_{2}\left(-\mathrm{I}_{1} / 2+\mathrm{g}_{82}+\mathrm{g}_{52}{ }^{2}\right)+\mathrm{g}_{84 \mathrm{~b}} \mathrm{w}_{4}+\mathrm{w}_{5}\left(\mathrm{~g}_{85 \mathrm{~b}}+\mathrm{g}_{52}\right)
$$

6.

$\mathrm{w}_{6} \mathrm{~S}+\mathrm{S} \mathrm{w}_{6}-2 / 3<\mathrm{w}_{6} \mathrm{~S}>\mathrm{I}=\mathrm{w}_{1}\left(-2 \mathrm{~g}_{31} \mathrm{~g}_{61}-2 \mathrm{~g}_{60}+2 \mathrm{~g}_{31}{ }^{2} \mathrm{~g}_{63 \mathrm{~b}}+2 \mathrm{~g}_{30} \mathrm{~g}_{63 \mathrm{~b}}+\right.$

$2 g_{31} g_{41} g_{64 b}+2 g_{40} g_{64 b}-2 g_{31}^{2} g_{43 b} g_{64 b}-2 g_{30} g_{43 b} g_{64 b}-g_{61} g_{64 b}-g_{91}+g_{41} I_{1}-$

$$
\left.\mathrm{g}_{63 \mathrm{~b}} \mathrm{I}_{1}+\mathrm{g}_{43 \mathrm{~b}} \mathrm{~g}_{64 \mathrm{~b}} \mathrm{I}_{1}+2 \mathrm{~g}_{31} \mathrm{I}_{2}+2 \mathrm{I}_{4}\right)+\mathrm{w}_{2}\left(-\mathrm{g}_{52}^{2} \mathrm{~g}_{65 \mathrm{~b}}-\mathrm{g}_{65 \mathrm{~b}} \mathrm{~g}_{82}-\mathrm{g}_{92}+\mathrm{g}_{65 \mathrm{~b}} \mathrm{I}_{1} / 2\right)+
$$

$w_{3}\left(-2 g_{61}+2 g_{31} g_{63 b}+2 g_{41} g_{64 b}-2 g_{31} g_{43 b} g_{64 b}-g_{63 b} g_{64 b}-g_{93 b}+g_{43 b} I_{1}+2 I_{2}\right)+$

$$
w_{4}\left(-g_{64 b}{ }^{2}-g_{65 b} g_{84 b}-g_{94 b}+I_{1}\right)+w_{5}\left(-g_{52} g_{65 b}-g_{64 b} g_{65 b}-g_{65 b} g_{85 b}-g_{95 b}\right)+w_{6}\left(-g_{64 b}-g_{96 b}\right)
$$

7.

$$
\mathrm{w}_{7} \mathrm{~S}+\mathrm{S} \mathrm{w}_{7}-2 / 3<\mathrm{w}_{7} \mathrm{~S}>\mathrm{I}=\mathrm{w}_{1}\left(-2 \mathrm{~g}_{101}+2 \mathrm{~g}_{31}{ }^{2} \mathrm{~g}_{73 \mathrm{~b}}+2 \mathrm{~g}_{30} \mathrm{~g}_{73 \mathrm{~b}}+\right.
$$

$\left.2 g_{31} g_{41} g_{74 b}+2 g_{40} g_{74 b}-2 g_{31}^{2} g_{43 b} g_{74 b}-2 g_{30} g_{43 b} g_{74 b}-g_{61} g_{74 b}-g_{73 b} I_{1}+g_{43 b} g_{74 b} I_{1}\right)+$

$\mathrm{w}_{2}\left(-2 \mathrm{~g}_{102}+\mathrm{g}_{52} \mathrm{~g}_{72}-\mathrm{g}_{52}^{2} \mathrm{~g}_{75 \mathrm{~b}}-\mathrm{g}_{75 \mathrm{~b}} \mathrm{~g}_{82}+\mathrm{g}_{75 \mathrm{~b}} \mathrm{I}_{1} / 2+\mathrm{g}_{52} \mathrm{I}_{2}-\mathrm{I}_{4}\right)+$

$\mathrm{w}_{3}\left(-2 \mathrm{~g}_{103 \mathrm{~b}}+2 \mathrm{~g}_{31} \mathrm{~g}_{73 \mathrm{~b}}+2 \mathrm{~g}_{41} \mathrm{~g}_{74 \mathrm{~b}}-2 \mathrm{~g}_{31} \mathrm{~g}_{43 \mathrm{~b}} \mathrm{~g}_{74 \mathrm{~b}}-\mathrm{g}_{63 \mathrm{~b}} \mathrm{~g}_{74 \mathrm{~b}}\right)+$

$\mathrm{w}_{4}\left(-2 \mathrm{~g}_{104 \mathrm{~b}}-\mathrm{g}_{64 \mathrm{~b}} \mathrm{~g}_{74 \mathrm{~b}}-\mathrm{g}_{75 \mathrm{~b}} \mathrm{~g}_{84 \mathrm{~b}}\right)+$

$w_{5}\left(-2 g_{105 b}+g_{72}-g_{65 b} g_{74 b}-g_{52} g_{75 b}-g_{75 b} g_{85 b}+I_{2}\right)+w_{6}\left(-g_{74 b}\right)+w_{7}\left(-2 g_{107 b}\right)$

To find the matrix $\mathrm{J}_{\mathrm{ij}}$, that is $[7 \times \mathrm{x} 7$ matrix

1.

$$
\mathrm{w}_{1} \mathrm{~W}-\mathrm{W} \mathrm{w}_{1}=\mathrm{S} \mathrm{W}-\mathrm{W} \mathrm{S}, \quad \mathrm{w}_{1} \mathrm{~W}-\mathrm{W} \mathrm{w}_{1}=\mathrm{w}_{2}
$$

2.

$\mathrm{W}_{2} \mathrm{~W}-\mathrm{W} \mathrm{w}_{2}=(\mathrm{S} \mathrm{W}-\mathrm{W} \mathrm{S}) \mathrm{W}-\mathrm{W}(\mathrm{S} \mathrm{W}-\mathrm{W} \mathrm{S})=\mathrm{S} \mathrm{W} \mathrm{W}^{2}-\mathrm{W} \mathrm{S} \mathrm{W}-\mathrm{W} \mathrm{S} \mathrm{W}+\mathrm{W}^{2} \mathrm{~S}$

where 


$$
\mathrm{S} \mathrm{W} \mathrm{W}^{2}+\mathrm{W}^{2} \mathrm{~S}=\mathrm{v}_{6}
$$

then

$$
=\mathrm{v}_{6}-2 \mathrm{~W} \mathrm{~S} \mathrm{~W}
$$

From equation (3.14), let $\mathrm{a}=\mathrm{W}, \mathrm{b}=\mathrm{S}$.

$$
\mathrm{W} \mathrm{S} \mathrm{W}=-\left(\mathrm{W}^{2} \mathrm{~S}+\mathrm{S} \mathrm{W}^{2}\right)+\mathrm{I}_{2} / 2 \mathrm{~S}+\mathrm{II}_{4}
$$

then

$$
\mathrm{w}_{2} \mathrm{~W}-\mathrm{W} \mathrm{w}_{2}=3 \mathrm{v}_{6}-\mathrm{I}_{2} \mathrm{~S}-2 \mathrm{I} \mathrm{I}_{4}
$$

where

$$
\mathrm{v}_{6}=\mathrm{w}_{6}+\mathrm{g}_{65 \mathrm{~b}} \mathrm{w}_{5}+\mathrm{g}_{64 \mathrm{~b}} \mathrm{w}_{4}+\mathrm{g}_{63 \mathrm{~b}} \mathrm{w}_{3}+\mathrm{g}_{61} \mathrm{w}_{1}+\mathrm{g}_{60} \mathrm{I} \text { and } \mathrm{S}=\mathrm{w}_{1}
$$

Thus,

3.

$$
\begin{gathered}
\mathrm{w}_{2} \mathrm{~W}-\mathrm{W} \mathrm{w}_{2}=\mathrm{w}_{1}\left(3 \mathrm{~g}_{61}-\mathrm{I}_{2}\right)+\mathrm{w}_{3}\left(3 \mathrm{~g}_{63 \mathrm{~b}}\right)+\mathrm{w}_{4}\left(3 \mathrm{~g}_{64 \mathrm{~b}}\right)+\mathrm{w}_{5}\left(3 \mathrm{~g}_{65 \mathrm{~b}}\right)+3 \mathrm{w}_{6} \\
\mathrm{w}_{3} \mathrm{~W}-\mathrm{W} \mathrm{w}_{3}=\left(\mathrm{v}_{3}-\mathrm{g}_{31} \mathrm{v}_{1}-\mathrm{g}_{30} \mathrm{I}\right) \mathrm{W}-\mathrm{W}\left(\mathrm{v}_{3}-\mathrm{g}_{31} \mathrm{v}_{1}-\mathrm{g}_{30} \mathrm{I}\right)
\end{gathered}
$$

Where

$$
\mathrm{v}_{3}=\mathrm{S}^{2}
$$

then

$$
=\mathrm{S}^{2} \mathrm{~W}-\mathrm{g}_{31} \mathrm{~S} \mathrm{~W}-\mathrm{g}_{30} \mathrm{I} \mathrm{W}-\mathrm{W} \mathrm{S}^{2}+\mathrm{g}_{31} \mathrm{~W} \mathrm{~S}+\mathrm{g}_{30} \mathrm{I} \mathrm{W}
$$

Where

$$
-\mathrm{g}_{31}(\mathrm{~S} W-\mathrm{W})=-\mathrm{g}_{31} \mathrm{v}_{2} \text {, and } \mathrm{S}^{2} \mathrm{~W}-\mathrm{W} \mathrm{S} \mathrm{S}^{2}=-\mathrm{v}_{5}=-\mathrm{w}_{5}-\mathrm{g}_{52} \mathrm{w}_{2}
$$

Thus,

$$
\mathrm{w}_{3} \mathrm{~W}-\mathrm{W} \mathrm{w}_{3}=\mathrm{w}_{2}\left(-\mathrm{g}_{52}-\mathrm{g}_{31}\right)-\mathrm{w}_{5}
$$

Similarly

4.

5.

$$
\begin{gathered}
\mathrm{w}_{4} \mathrm{~W}-\mathrm{W} \mathrm{w}_{4}=\mathrm{w}_{2}\left(\mathrm{~g}_{43 \mathrm{~b}} \mathrm{~g}_{52}+\mathrm{g}_{43 \mathrm{~b}} \mathrm{~g}_{31}-\mathrm{g}_{41}\right)+\mathrm{w}_{5} \mathrm{~g}_{43 \mathrm{~b}} \\
\mathrm{w}_{5} \mathrm{~W}-\mathrm{W} \mathrm{w}_{5}=\mathrm{w}_{1}\left(-3 \mathrm{~g}_{91}-3 \mathrm{~g}_{52} \mathrm{~g}_{61}+\mathrm{g}_{52} \mathrm{I}_{2}+\mathrm{I}_{2} \mathrm{~g}_{31}+2 \mathrm{I}_{1} \mathrm{~g}_{41}\right)+\mathrm{w}_{2}\left(-3 \mathrm{~g}_{92}\right)+ \\
\mathrm{w}_{3}\left(-3 \mathrm{~g}_{52} \mathrm{~g}_{63 \mathrm{~b}}+\mathrm{I}_{2}+2 \mathrm{I}_{1} \mathrm{~g}_{43 \mathrm{~b}}-3 \mathrm{~g}_{93 \mathrm{~b}}\right)+\mathrm{w}_{4}\left(-3 \mathrm{~g}_{94 \mathrm{~b}}-3 \mathrm{~g}_{52} \mathrm{~g}_{64 \mathrm{~b}}+2 \mathrm{I}_{1}\right)+ \\
\mathrm{w}_{5}\left(-3 \mathrm{~g}_{95 \mathrm{~b}}-3 \mathrm{~g}_{52} \mathrm{~g}_{65 \mathrm{~b}}\right)+\mathrm{w}_{6}\left(-3 \mathrm{~g}_{96 \mathrm{~b}}-3 \mathrm{~g}_{52}\right)
\end{gathered}
$$

6.

$$
\begin{aligned}
\mathrm{w}_{6} \mathrm{~W}-\mathrm{W} \mathrm{w}_{6}= & \mathrm{w}_{1}\left(3 \mathrm{~g}_{52} \mathrm{~g}_{61} \mathrm{~g}_{65 \mathrm{~b}}+3 \mathrm{~g}_{65 \mathrm{~b}} \mathrm{~g}_{91}-2 \mathrm{~g}_{41} \mathrm{~g}_{65 \mathrm{~b}} \mathrm{I}_{1}-\mathrm{g}_{31} \mathrm{~g}_{65 \mathrm{~b}} \mathrm{I}_{2}-\mathrm{g}_{52} \mathrm{~g}_{65 \mathrm{~b}} \mathrm{I}_{2}\right)+ \\
& \mathrm{w}_{2}\left(-\mathrm{g}_{61}+\mathrm{g}_{31} \mathrm{~g}_{63 \mathrm{~b}}+\mathrm{g}_{52} \mathrm{~g}_{63 \mathrm{~b}}+\mathrm{g}_{41} \mathrm{~g}_{64 \mathrm{~b}}-\mathrm{g}_{31} \mathrm{~g}_{43 \mathrm{~b}} \mathrm{~g}_{64 \mathrm{~b}}-\mathrm{g}_{43 \mathrm{~b}} \mathrm{~g}_{52} \mathrm{~g}_{64 \mathrm{~b}}-\mathrm{g}_{72}+3 \mathrm{~g}_{65 \mathrm{~b}} \mathrm{~g}_{92}+\mathrm{I}_{2} / 2\right)+ \\
& \mathrm{w}_{3}\left(3 \mathrm{~g}_{52} \mathrm{~g}_{63 \mathrm{~b}} \mathrm{~g}_{65 \mathrm{~b}}-\mathrm{g}_{73 \mathrm{~b}}+3 \mathrm{~g}_{65 \mathrm{~b}} \mathrm{~g}_{93 \mathrm{~b}}-2 \mathrm{~g}_{43 \mathrm{~b}} \mathrm{~g}_{65 \mathrm{~b}} \mathrm{I}_{1}-\mathrm{g}_{65 \mathrm{~b}} \mathrm{I}_{2}\right)+ \\
& \mathrm{w}_{4}\left(3 \mathrm{~g}_{52} \mathrm{~g}_{64 \mathrm{~b}} \mathrm{~g}_{65 \mathrm{~b}}-\mathrm{g}_{74 \mathrm{~b}}+3 \mathrm{~g}_{65 \mathrm{~b}} \mathrm{~g}_{94 \mathrm{~b}}-2 \mathrm{~g}_{65 \mathrm{~b}} \mathrm{I}_{1}\right)+ \\
& \mathrm{w}_{5}\left(\mathrm{~g}_{63 \mathrm{~b}}-\mathrm{g}_{43 \mathrm{~b}} \mathrm{~g}_{64 \mathrm{~b}}+3 \mathrm{~g}_{52} \mathrm{~g}_{65 \mathrm{~b}}{ }^{2}-\mathrm{g}_{75 \mathrm{~b}}+3 \mathrm{~g}_{65 \mathrm{~b}} \mathrm{~g}_{95 \mathrm{~b}}\right)+ \\
& \mathrm{w}_{6}\left(3 \mathrm{~g}_{52} \mathrm{~g}_{65 \mathrm{~b}}+3 \mathrm{~g}_{65 \mathrm{~b}} \mathrm{~g}_{96 \mathrm{~b}}\right)-\mathrm{w}_{7}
\end{aligned}
$$

7.

$$
\begin{gathered}
\mathrm{w}_{7} \mathrm{~W}-\mathrm{W} \mathrm{w}_{7}=\mathrm{w}_{1}\left(-3 \mathrm{~g}_{61} \mathrm{~g}_{72}+3 \mathrm{~g}_{52} \mathrm{~g}_{61} \mathrm{~g}_{75 \mathrm{~b}}+3 \mathrm{~g}_{75 \mathrm{~b}} \mathrm{~g}_{91}-2 \mathrm{~g}_{41} \mathrm{~g}_{75 \mathrm{~b}} \mathrm{I}_{1}-2 \mathrm{~g}_{61} \mathrm{I}_{2}+\right. \\
\left.\mathrm{g}_{72} \mathrm{I}_{2}-\mathrm{g}_{31} \mathrm{~g}_{75 \mathrm{~b}} \mathrm{I}_{2}-\mathrm{g}_{52} \mathrm{~g}_{75 \mathrm{~b}} \mathrm{I}_{2}+\mathrm{I}_{2}^{2}-2 \mathrm{~g}_{41} \mathrm{I}_{4}\right)+ \\
\mathrm{w}_{2}\left(\mathrm{~g}_{31} \mathrm{~g}_{73 \mathrm{~b}}+\mathrm{g}_{52} \mathrm{~g}_{73 \mathrm{~b}}+\mathrm{g}_{52} \mathrm{~g}_{73 \mathrm{~b}}+\mathrm{g}_{41} \mathrm{~g}_{74 \mathrm{~b}}-\mathrm{g}_{31} \mathrm{~g}_{43 \mathrm{~b}} \mathrm{~g}_{74 \mathrm{~b}}-\mathrm{g}_{43 \mathrm{~b}} \mathrm{~g}_{52} \mathrm{~g}_{74 \mathrm{~b}}+3 \mathrm{~g}_{75 \mathrm{~b}} \mathrm{~g}_{92}\right)+ \\
\mathrm{w}_{3}\left(-3 \mathrm{~g}_{63 \mathrm{~b}} \mathrm{~g}_{72}+3 \mathrm{~g}_{52} \mathrm{~g}_{63 \mathrm{~b}} \mathrm{~g}_{75 \mathrm{~b}}+3 \mathrm{~g}_{75 \mathrm{~b}} \mathrm{~g}_{93 \mathrm{~b}}-2 \mathrm{~g}_{43 \mathrm{~b}} \mathrm{~g}_{75 \mathrm{~b}} \mathrm{I}_{1}-2 \mathrm{~g}_{63 \mathrm{~b}} \mathrm{I}_{2}-\mathrm{g}_{75 \mathrm{~b}} \mathrm{I}_{2}-2 \mathrm{~g}_{43 \mathrm{~b}} \mathrm{I}_{4}\right)+ \\
\mathrm{w}_{4}\left(-3 \mathrm{~g}_{64 \mathrm{~b}} \mathrm{~g}_{72}+3 \mathrm{~g}_{52} \mathrm{~g}_{64 \mathrm{~b}} \mathrm{~g}_{75 \mathrm{~b}}+3 \mathrm{~g}_{75 \mathrm{~b}} \mathrm{~g}_{94 \mathrm{~b}}-2 \mathrm{~g}_{75 \mathrm{~b}} \mathrm{I}_{1}-2 \mathrm{~g}_{64 \mathrm{~b}} \mathrm{I}_{2}-2 \mathrm{I}_{4}\right)+ \\
\mathrm{w}_{5}\left(-3 \mathrm{~g}_{65 \mathrm{~b}} \mathrm{~g}_{72}+\mathrm{g}_{73 \mathrm{~b}}-\mathrm{g}_{43 \mathrm{~b}} \mathrm{~g}_{74 \mathrm{~b}}+3 \mathrm{~g}_{52} \mathrm{~g}_{65 \mathrm{~b}} \mathrm{~g}_{75 \mathrm{~b}}+3 \mathrm{~g}_{75 \mathrm{~b}} \mathrm{~g}_{95 \mathrm{~b}}-2 \mathrm{~g}_{65 \mathrm{~b}} \mathrm{I}_{2}\right)+ \\
\mathrm{w}_{6}\left(-3 \mathrm{~g}_{72}+3 \mathrm{~g}_{52} \mathrm{~g}_{75 \mathrm{~b}}+3 \mathrm{~g}_{75 \mathrm{~b}} \mathrm{~g}_{96 \mathrm{~b}}-2 \mathrm{I}_{2}\right)
\end{gathered}
$$

To find the matrix $\mathrm{A}_{\mathrm{ij}}=-\delta_{\mathrm{ji}}-\mathrm{H}_{\mathrm{ji}}+\mathrm{J}_{\mathrm{ji}}$.

The main issue in obtaining an explicit relation for the anisotropy is determining the $\mathrm{L}_{\mathrm{i}}$ coefficients by taking the inverse of the matrix $A_{i j}$, the first column of the matrix $A_{i j}$ are the solutions of $\mathrm{L}_{1}, \mathrm{~L}_{2}, \mathrm{~L}_{3}, \mathrm{~L}_{4}, \mathrm{~L}_{5}, \mathrm{~L}_{6}$, and $\mathrm{L}_{7}$. In mathematical computation can be used by software Mathematica version 4.0 to calculate all this research, the matrices $\mathrm{A}_{\mathrm{ij}}, \mathrm{H}_{\mathrm{ji}}$, and $\mathrm{J}_{\mathrm{ji}}$. 


\section{Method Two: Calculation of the Anisotropy Tensor}

Using equations (3.19) to (3.20) to calculate the anisotropy tensor and compare the present results with the results of Gatski and Speziale, they let the anisotropy tensor as is function of ten terms $T_{i}$ that are the integrity basis for functions of symmetric and antisymmetric tensor, and ten $\mathrm{G}_{\mathrm{i}}$ are scalar functions of the irreducible invariants of $S_{i j}$ and $\mathrm{W}_{\mathrm{ij}}$ as follows:

$$
\begin{gathered}
\mathrm{b}_{\mathrm{ij}}=\sum_{i=1}^{10} \mathrm{G}_{\mathrm{i}} \mathrm{T}_{\mathrm{i}} \\
=\sum_{i=1}^{7} \mathrm{G}_{\mathrm{i}} \mathrm{T}_{\mathrm{i}}+\sum_{i=8}^{10} \mathrm{G}_{\mathrm{i}} \mathrm{T}_{\mathrm{i}}
\end{gathered}
$$

From equation (3.22) the expression between $\mathrm{w}_{\mathrm{i}}$ and $\mathrm{T}_{\mathrm{i}}$ can be written as

$$
\begin{aligned}
& \sum_{i=1}^{7} \mathrm{w}_{\mathrm{i}}=\left[\mathrm{P}_{\mathrm{ij}}\right] \sum_{j=1}^{7} \mathrm{~T}_{\mathrm{j}} \\
& \sum_{j=1}^{7} \mathrm{~T}_{\mathrm{j}}=\left[\mathrm{P}_{\mathrm{ij}}\right]^{-1} \sum_{i=1}^{7} \mathrm{w}_{\mathrm{i}}
\end{aligned}
$$

and from equation (3.24) the expression between $T_{i}$ and $w_{i}$ can be written as

$$
\sum_{i=8}^{10} \mathrm{~T}_{\mathrm{i}}=\left[\mathrm{Q}_{\mathrm{ij}}\right] \sum_{j=1}^{7} \mathrm{w}_{\mathrm{j}}
$$

From equations (3.24) to (3.25) lead to find the anisotropy tensor $b_{i j}$ that is result to compare the present solution with Gastki and Speziale's solution (p. 45).

$$
\mathrm{b}_{\mathrm{ij}}=\left[\mathrm{G}_{1}, \mathrm{G}_{2}, \mathrm{G}_{3}, \mathrm{G}_{4}, \mathrm{G}_{5}, \mathrm{G}_{6}, \mathrm{G}_{7}\right]\left[\mathrm{P}_{\mathrm{ij}}\right]^{-1} \sum_{i=1}^{7} \mathrm{w}_{\mathrm{i}}+\left[\mathrm{G}_{8}, \mathrm{G}_{9}, \mathrm{G}_{10}\right]\left[\mathrm{Q}_{\mathrm{ij}}\right] \sum_{j=1}^{7} \mathrm{w}_{\mathrm{j}}
$$

Thus,

$$
\begin{gathered}
b_{i j}=\left(G_{1}+G_{10} g_{101}+G_{3} g_{31}+G_{4} g_{41}+G_{6} g_{61}+G_{9} g_{91}\right) w_{1}+ \\
\left(G_{10} g_{102}+G_{2}+G_{5} g_{52}+G_{7} g_{72}+G_{8} g_{82}+G_{9} g_{92}\right) w_{2}+ \\
\left(G_{10} g_{103 b}+G_{3}+G_{4} g_{43 b}+G_{6} g_{63 b}+G_{7} g_{73 b}+G_{9} g_{93 b}\right) w_{3}+ \\
\left(G_{10} g_{104 b}+G_{4}+G_{6} g_{64 b}+G_{7} g_{74 b}+G_{8} g_{84 b}+G_{9} g_{94 b}\right) w_{4}+ \\
\left(G_{10} g_{105 b}+G_{5}+G_{6} g_{65 b}+G_{7} g_{75 b}+G_{8} g_{85 b}+G_{9} g_{95 b}\right) w_{5}+ \\
\left(G_{6}+G_{9} g_{96 b}\right) w_{6}+\left(G_{10} g_{107 b}+G_{7}\right) w_{7}
\end{gathered}
$$

where

$$
\begin{gathered}
\mathrm{g}_{101}=\frac{\operatorname{trace}\left(v_{10} \cdot v_{1}\right)}{\operatorname{trace}\left(v_{1} \cdot v_{1}\right)} \quad \text { and } \quad \mathrm{g}_{43 \mathrm{~b}}=\frac{\operatorname{trace}\left(v_{4} \cdot w_{3}\right)}{\operatorname{trace}\left(w_{3} \cdot w_{3}\right)} \\
\mathrm{v}_{10} \cdot \mathrm{W}_{1}=\left(\mathrm{W} \mathrm{S}^{2} \mathrm{~W}^{2}-\mathrm{W}^{2} \mathrm{~S}^{2} \mathrm{~W}\right) . \mathrm{S}=\mathrm{W} \mathrm{S}^{2} \mathrm{~W}^{2} \mathrm{~S}-\mathrm{W}^{2} \mathrm{~S}^{2} \mathrm{~W} \mathrm{~S}
\end{gathered}
$$

then

$$
\operatorname{trace}\left(\mathrm{v}_{10} \cdot \mathrm{W}_{1}\right)=\operatorname{trace}\left(\mathrm{W} \mathrm{S}^{2} \mathrm{~W}^{2} \mathrm{~S}\right)-\operatorname{trace}\left(\mathrm{W}^{2} \mathrm{~S}^{2} \mathrm{~W} \mathrm{~S}\right)
$$

and from equation (3.27), let $\mathrm{a}=\mathrm{W}, \mathrm{b}=\mathrm{S}$ then

$$
\mathrm{W} \mathrm{S} \mathrm{W}^{2}+\mathrm{W}^{2} \mathrm{~S} \mathrm{~W}=\mathrm{W} \mathrm{S} \mathrm{W} \operatorname{trace}(\mathrm{W})+\mathrm{W}^{2} \operatorname{trace}(\mathrm{W} \mathrm{S})+
$$

$\mathrm{W}\left[\operatorname{trace}\left(\mathrm{W}^{2} \mathrm{~S}\right)-\operatorname{trace}(\mathrm{W}) \operatorname{trace}(\mathrm{W} S)\right]-\mathrm{S} \operatorname{determinant}(\mathrm{W})+\mathrm{I} \operatorname{determinant}(\mathrm{W}) \operatorname{trace}(\mathrm{S})$ 
Where

$$
\operatorname{trace}(\mathrm{W})=0, \operatorname{trace}(\mathrm{W} \mathrm{S})=0, \operatorname{trace}(\mathrm{S})=0
$$

and

$$
\operatorname{determinant}(\mathrm{W})=0
$$

then

$$
\mathrm{W} \mathrm{S} \mathrm{W}^{2}+\mathrm{W}^{2} \mathrm{~S} \mathrm{~W}=\mathrm{W} \operatorname{trace}\left(\mathrm{W}^{2} \mathrm{~S}\right)
$$

Where

$$
\operatorname{trace}\left(\mathrm{W}^{2} \mathrm{~S}\right)=\operatorname{trace}\left(\mathrm{S} \mathrm{W}^{2}\right)=\mathrm{I}_{4}
$$

Thus, equation (3.28) becomes

$$
\mathrm{W} \mathrm{S} \mathrm{W} \mathrm{W}^{2}+\mathrm{W}^{2} \mathrm{~S} \mathrm{~W}=\mathrm{W} \mathrm{I}_{4}
$$

Multiplication the left side and the right side of equation (3.29) with $S^{2}$ then

$$
\mathrm{S}^{2} \mathrm{~W} \mathrm{~S} \mathrm{~W}^{2}+\mathrm{S}^{2} \mathrm{~W}^{2} \mathrm{~S} \mathrm{~W}=\mathrm{I}_{4} \mathrm{~S}^{2} \mathrm{~W}
$$

Taking the trace for both side of equation (3.30) then

$$
\operatorname{trace}\left(\mathrm{S}^{2} \mathrm{~W} \mathrm{~S} \mathrm{~W}^{2}\right)+\operatorname{trace}\left(\mathrm{S}^{2} \mathrm{~W}^{2} \mathrm{~S} \mathrm{~W}\right)=\mathrm{I}_{4} \operatorname{trace}\left(\mathrm{S}^{2} \mathrm{~W}\right)
$$

where

thus equation (3.31) yields

$$
\operatorname{trace}\left(\mathrm{S}^{2} \mathrm{~W}\right)=0 \text { and } \operatorname{trace}\left(\mathrm{S}^{2} \mathrm{~W}^{2} \mathrm{~S} \mathrm{~W}\right)=\mathrm{I}_{6}
$$

$$
-\operatorname{trace}\left(S^{2} W_{S ~ W}^{2}\right)=\operatorname{trace}\left(S^{2} W^{2} S W\right)=I_{6}
$$

Then

$$
\begin{gathered}
\operatorname{trace}\left(\mathrm{v}_{10} \cdot \mathrm{w}_{1}\right)=\operatorname{trace}\left(\mathrm{W} \mathrm{S}^{2} \mathrm{~W}^{2} \mathrm{~S}\right)-\operatorname{trace}\left(\mathrm{W}^{2} \mathrm{~S}^{2} \mathrm{~W} \mathrm{~S}\right)=2 \mathrm{I}_{6} \\
\operatorname{trace}\left(\mathrm{w}_{1} \cdot \mathrm{w}_{1}\right)=\operatorname{trace}(\mathrm{S} . \mathrm{S})=\mathrm{I}_{1}
\end{gathered}
$$

Thus

$$
\begin{gathered}
\mathrm{g}_{101}=2 \mathrm{I}_{6} / \mathrm{I}_{1} \\
\mathrm{~g}_{31}=\frac{\operatorname{trace}\left(v_{3} \cdot w_{1}\right)}{\operatorname{trace}\left(w_{1} \cdot w_{1}\right)}=\frac{\operatorname{trace}\left(S^{2} \cdot S\right)}{\operatorname{trace}(S \cdot S)}=\frac{I_{3}}{I_{1}} \\
\mathrm{~g}_{41}=\frac{\operatorname{trace}\left(v_{4} \cdot w_{1}\right)}{\operatorname{trace}\left(w_{1} \cdot w_{1}\right)}=\frac{\operatorname{trace}\left(W^{2} \cdot S\right)}{\operatorname{trace}(S \cdot S)}=\frac{I_{4}}{I_{1}} \\
\mathrm{~g}_{61}=\frac{\operatorname{trace}\left(v_{6} \cdot w_{1}\right)}{\operatorname{trace}\left(w_{1} \cdot w_{1}\right)}=\frac{\operatorname{trace}\left(S W^{2}+W^{2} S\right) S}{\operatorname{trace}(S \cdot S)}=\frac{2 \operatorname{trace}\left(S^{2} W^{2}\right)}{I_{1}}=\frac{2 I_{5}}{I_{1}} \\
g_{91}=\frac{\operatorname{trace}\left(v_{9} \cdot w_{1}\right)}{\operatorname{trace}\left(w_{1} \cdot w_{1}\right)}=\frac{\operatorname{trace}\left(W^{2} S^{2}+S^{2} W^{2}\right) S}{\operatorname{trace}(S \cdot S)}=\frac{\operatorname{trace}\left(W^{2} S^{3}+S^{3} W^{2}\right)}{I_{1}}=\frac{I_{1} I_{4}+(2 / 3) I_{2} I_{3}}{I_{1}}
\end{gathered}
$$

Similarly

$$
\begin{aligned}
& \mathrm{g}_{43 \mathrm{~b}}=\frac{\operatorname{trace}\left(v_{4} \cdot w_{3}\right)}{\operatorname{trace}\left(w_{3} \cdot w_{3}\right)}=\left(I_{5}-I_{3} \frac{I_{4}}{I_{1}}-I_{1} \frac{I_{2}}{3}\right) /\left(\frac{1}{6} I_{1}^{2}-I_{3}^{2} / I_{1}\right) \\
& \mathrm{g}_{102 \mathrm{~b}}=\frac{\operatorname{trace}\left(v_{10} \cdot w_{2}\right)}{\operatorname{trace}\left(w_{2} \cdot w_{2}\right)}=\frac{I_{2}{ }^{2} I_{3} / 3+2 I_{4} I_{5}}{I_{1} I_{2}-6 I_{5}} \\
& \mathrm{~g}_{52}=\frac{\operatorname{trace}\left(v_{5} \cdot w_{2}\right)}{\operatorname{trace}\left(w_{2} \cdot w_{2}\right)}=\frac{I_{1} I_{4}+I_{2} I_{3}}{I_{1} I_{2}-6 I_{5}} \\
& \mathrm{~g}_{63 \mathrm{~b}}=\frac{\operatorname{trace}\left(v_{6} \cdot w_{3}\right)}{\operatorname{trace}\left(w_{3} \cdot w_{3}\right)}=\left(\frac{1}{3} I_{1} I_{4}+\frac{2}{3} I_{2} I_{3}-2 I_{3} I_{5} / I_{1}\right) /\left(\frac{1}{6} I_{1}^{2}-I_{3}^{2} / I_{1}\right)
\end{aligned}
$$




$$
\begin{aligned}
& \mathrm{g}_{73 \mathrm{~b}}=\frac{\operatorname{trace}\left(v_{7} \cdot w_{3}\right)}{\operatorname{trace}\left(w_{3} \cdot w_{3}\right)}=-2 I_{6} /\left(\frac{1}{6} I_{1}^{2}-I_{3}^{2} / I_{1}\right) \\
& \mathrm{g}_{93 \mathrm{~b}}=\frac{\operatorname{trace}\left(v_{9} \cdot w_{3}\right)}{\operatorname{trace}\left(w_{3} \cdot w_{3}\right)}=\left(I_{1} I_{5}-I_{3} I_{4} / 3-\frac{2}{3} I_{2} \frac{I_{3}^{2}}{I_{1}}-\frac{2}{3} I_{1} I_{5}\right) /\left(\frac{1}{6} I_{1}^{2}-I_{3}^{2} / I_{1}\right) \\
& \mathrm{g}_{103 \mathrm{~b}}=\frac{\operatorname{trace}\left(v_{10} \cdot w_{3}\right)}{\operatorname{trace}\left(w_{3} \cdot w_{3}\right)}=-\frac{2 I_{3} I_{6}}{I_{1}} \\
& \mathrm{~g}_{43 \mathrm{~b}}=\frac{\operatorname{trace}\left(v_{4} \cdot w_{3}\right)}{\operatorname{trace}\left(w_{3} \cdot w_{3}\right)}=\left(I_{5}-I_{3} \frac{I_{4}}{I_{1}}-I_{1} \frac{I_{2}}{3}\right) /\left(\frac{1}{6} I_{1}^{2}-I_{3}^{2} / I_{1}\right)
\end{aligned}
$$

\section{Discussion of the Numerical Results}

\section{Comparison of the Numerical Results the Anisotropy Tensor}

The mean of strain rate tensor $\mathrm{S}_{\mathrm{ij}}$ and the mean of rotation rate tensor $\mathrm{W}_{\mathrm{ij}}$ that the symmetric and antisymmetric parts of the mean velocity gradient tensor as

$$
\begin{gathered}
S_{i j}=\frac{1}{2}\left(\frac{\partial U_{i}}{\partial x_{j}}+\frac{\partial U_{j}}{\partial x_{i}}\right) \\
\mathrm{S}_{\mathrm{ij}}=\left|\begin{array}{ccc}
\mathrm{s}_{\mathrm{a}} & \mathrm{s}_{12} & \mathrm{~s}_{13} \\
\mathrm{~s}_{21} & \mathrm{~s}_{\mathrm{b}} & \mathrm{s}_{23} \\
\mathrm{~s}_{31} & \mathrm{~s}_{32} & \mathrm{~s}_{\mathrm{c}}
\end{array}\right|
\end{gathered}
$$

where

$$
\begin{gathered}
\mathrm{s}_{\mathrm{a}}+\mathrm{s}_{\mathrm{b}}+\mathrm{s}_{\mathrm{c}}=0 \text { and } \mathrm{s}_{12}=\mathrm{s}_{21}, \mathrm{~s}_{13}=\mathrm{s}_{31}, \mathrm{~s}_{23}=\mathrm{s}_{32} \\
\mathrm{~W}_{\mathrm{ij}}=\left|\begin{array}{lll}
0 & \mathrm{w}_{\mathrm{a}} & \mathrm{w}_{\mathrm{b}} \\
\mathrm{w}_{\mathrm{a}} & 0 & \mathrm{w}_{\mathrm{c}} \\
\mathrm{w}_{\mathrm{b}} & \mathrm{w}_{\mathrm{c}} & 0
\end{array}\right|
\end{gathered}
$$

Mathematica version 4.0 (Wolfram, 1999) was used to determine all the mathematical computation in this research. The following describes the steps of performing the computation calculations. First input any random numbers of $\mathrm{S}_{\mathrm{ij}}$ and $\mathrm{W}_{\mathrm{ij}}$ from -1. to 1. and check the numbers of $\mathrm{w}_{1}, \mathrm{w}_{2}, \mathrm{w}_{3}, \mathrm{w}_{4}, \mathrm{w}_{5}, \mathrm{w}_{6}$, and $\mathrm{w}_{7}$, if one or more of them equal to zero, for instance, if $\mathrm{w}_{3}=\{\{0 ., 0 ., 0\},.\{0 ., 0 ., 0\},.\{0 ., 0 ., 0\}$.$\} and \mathrm{w}_{5}=\{\{0 ., 0 ., 0\},.\{0 ., 0 ., 0\},.\{0 ., 0 ., 0\}$.$\} then$ $\mathrm{g}_{43 \mathrm{~b}}, \mathrm{~g}_{63 \mathrm{~b}}, \mathrm{~g}_{65 \mathrm{~b}}, \mathrm{~g}_{73 \mathrm{~b}}, \mathrm{~g}_{75 \mathrm{~b}}, \mathrm{~g}_{85 \mathrm{~b}}, \mathrm{~g}_{93 \mathrm{~b}}, \mathrm{~g}_{95 \mathrm{~b}}, \mathrm{~g}_{103 \mathrm{~b}}$, and $\mathrm{g}_{105 \mathrm{~b}}$ will approach some constant and after that these terms time with $\mathrm{w}_{3}$ and $\mathrm{w}_{5}$ then they always equal to zero, it means every g relates to $\mathrm{w}_{3}=\{\{0 ., 0 ., 0\},.\{0 ., 0 ., 0\},.\{0 ., 0 ., 0\}$. and $\mathrm{w}_{5}=\{\{0 ., 0 ., 0\},.\{0 ., 0 ., 0\},.\{0 ., 0 ., 0\}$.$\} have to set equal to some constant because \mathrm{g}_{43 \mathrm{~b}}=\operatorname{trace}\left(\mathrm{v}_{4} \mathrm{w}_{3}\right) / \operatorname{trace}\left(\mathrm{w}_{3}\right.$ $\mathrm{w}_{3}$ ) that Mathematica could not solve but actual $\mathrm{w}_{3}$ and $\mathrm{w}_{5}$ approaches very small constants, then the program is ran again. The result of the anisotropy tensor $b_{\mathrm{ij}}$ can be written as follows:

$$
\begin{aligned}
b_{i j}= & \left(G_{1}+G_{10} g_{101}+G_{3} g_{31}+G_{4} g_{41}+G_{6} g_{61}+G_{9} g_{91}\right) w_{1}+ \\
& \left(G_{10} g_{102}+G_{2}+G_{5} g_{52}+G_{7} g_{72}+G_{8} g_{82}+G_{9} g_{92}\right) w_{2}+ \\
& \left(G_{10} g_{103 b}+G_{3}+G_{4} g_{43 b}+G_{6} g_{63 b}+G_{7} g_{73 b}+G_{9} g_{93 b}\right) w_{3}+ \\
& \left(G_{10} g_{104 b}+G_{4}+G_{6} g_{64 b}+G_{7} g_{74 b}+G_{8} g_{84 b}+G_{9} g_{94 b}\right) w_{4}+ \\
& \left(G_{10} g_{105 b}+G_{5}+G_{6} g_{65 b}+G_{7} g_{75 b}+G_{8} g_{85 b}+G_{9} g_{95 b}\right) w_{5}+ \\
& \left(G_{6}+G_{9} g_{96 b}\right) w_{6}+\left(G_{10} g_{107 b}+G_{7}\right) w_{7}
\end{aligned}
$$

and $\mathrm{w}_{3}=\{\{0 ., 0 ., 0\},.\{0 ., 0 ., 0\},.\{0 ., 0 ., 0\}\},. \mathrm{w}_{5}=\{\{0 ., 0 ., 0\},.\{0 ., 0 ., 0\},.\{0 ., 0 ., 0\}$.$\} then these terms as:$

$$
\begin{aligned}
& \left(\mathrm{G}_{10} \mathrm{~g}_{103 \mathrm{~b}}+\mathrm{G}_{3}+\mathrm{G}_{4} \mathrm{~g}_{43 \mathrm{~b}}+\mathrm{G}_{6} \mathrm{~g}_{63 \mathrm{~b}}+\mathrm{G}_{7} \mathrm{~g}_{73 \mathrm{~b}}+\mathrm{G}_{9} \mathrm{~g}_{93 \mathrm{~b}}\right) \mathrm{w}_{3}=\{\{0 ., 0 ., 0 .\},\{0 ., 0 ., 0 .\},\{0 ., 0 ., 0 .\}\} \\
& \left(\mathrm{G}_{10} \mathrm{~g}_{105 \mathrm{~b}}+\mathrm{G}_{5}+\mathrm{G}_{6} \mathrm{~g}_{65 \mathrm{~b}}+\mathrm{G}_{7} \mathrm{~g}_{75 \mathrm{~b}}+\mathrm{G}_{8} \mathrm{~g}_{85 \mathrm{~b}}+\mathrm{G}_{9} \mathrm{~g}_{95 \mathrm{~b}}\right) \mathrm{w}_{5}=\{\{0 ., 0 ., 0 .\},\{0 ., 0 ., 0 .\},\{0 ., 0 ., 0 .\}\}
\end{aligned}
$$

Case 1:

$$
\mathrm{w}_{6}=\mathbf{0} \text { and } \mathrm{w}_{7}=\mathbf{0} \text { when } \mathrm{s}_{\mathrm{a}} \neq \mathrm{s}_{\mathrm{b}} \neq \mathrm{s}_{\mathrm{c}} \text { and } \mathrm{w}_{\mathrm{a}} \neq \mathrm{w}_{\mathrm{b}} \neq \mathrm{w}_{\mathrm{c}}
$$

where

$$
\mathbf{0}=\{\{0 ., 0 ., 0 .\},\{0 ., 0 ., 0 .,\},\{0 ., 0 ., 0 .\}\}
$$

Enter any random numbers of $\mathrm{S}_{\mathrm{ij}}$ and $\mathrm{W}_{\mathrm{ij}}$ from $[-1,1]$ as follows: 


$$
\begin{gathered}
\mathrm{S}_{\mathrm{ij}}=\left|\begin{array}{ccc}
0.55 & 0.0 & 0.0 \\
0.0 & 0.45 & 0.0 \\
0.0 & 0.4 & 0.6
\end{array}\right| \\
\mathrm{W}_{\mathrm{ij}=}=\left|\begin{array}{lll}
0.0 & 0.4 & 0.6 \\
0.6 & 0.7 & 0.0 \\
0.6 & 0.7 & 0.0
\end{array}\right|
\end{gathered}
$$

1. Using the method two to calculate the anisotropy tensor, then find the numerical results as

$$
\begin{aligned}
\mathrm{b}_{\mathrm{ij}}= & \left(\mathrm{G}_{1}+\mathrm{G}_{10} \mathrm{~g}_{101}+\mathrm{G}_{3} \mathrm{~g}_{31}+\mathrm{G}_{4} \mathrm{~g}_{41}+\mathrm{G}_{6} \mathrm{~g}_{61}+\mathrm{G}_{9} \mathrm{~g}_{91}\right) \mathrm{w}_{1}+ \\
& \left(\mathrm{G}_{10} \mathrm{~g}_{102}+\mathrm{G}_{2}+\mathrm{G}_{5} \mathrm{~g}_{52}+\mathrm{G}_{7} \mathrm{~g}_{72}+\mathrm{G}_{8} \mathrm{~g}_{82}+\mathrm{G}_{9} \mathrm{~g}_{92}\right) \mathrm{w}_{2}+ \\
& \left(\mathrm{G}_{10} \mathrm{~g}_{103 \mathrm{~b}}+\mathrm{G}_{3}+\mathrm{G}_{4} \mathrm{~g}_{43 \mathrm{~b}}+\mathrm{G}_{6} \mathrm{~g}_{63 \mathrm{~b}}+\mathrm{G}_{7} \mathrm{~g}_{73 \mathrm{~b}}+\mathrm{G}_{9} \mathrm{~g}_{93 \mathrm{~b}}\right) \mathrm{w}_{3}+ \\
& \left(\mathrm{G}_{10} \mathrm{~g}_{104 \mathrm{~b}}+\mathrm{G}_{4}+\mathrm{G}_{6} \mathrm{~g}_{64 \mathrm{~b}}+\mathrm{G}_{7} \mathrm{~g}_{74 \mathrm{~b}}+\mathrm{G}_{8} \mathrm{~g}_{84 \mathrm{~b}}+\mathrm{G}_{9} \mathrm{~g}_{94 \mathrm{~b}}\right) \mathrm{w}_{4}+ \\
& \left(\mathrm{G}_{10} \mathrm{~g}_{105 \mathrm{~b}}+\mathrm{G}_{5}+\mathrm{G}_{6} \mathrm{~g}_{65 \mathrm{~b}}+\mathrm{G}_{7} \mathrm{~g}_{75 \mathrm{~b}}+\mathrm{G}_{8} \mathrm{~g}_{85 \mathrm{~b}}+\mathrm{G}_{9} \mathrm{~g}_{95 \mathrm{~b}}\right) \mathrm{w}_{5}
\end{aligned}
$$

and the numerical results of $\mathrm{L}_{1}, \mathrm{~L}_{2}, \mathrm{~L}_{3}, \mathrm{~L}_{4}$, and $\mathrm{L}_{5}$ as follows:

$$
\begin{aligned}
& \mathrm{L}_{1}=\mathrm{G}_{1}+\mathrm{G}_{10} \mathrm{~g}_{101}+\mathrm{G}_{3} \mathrm{~g}_{31}+\mathrm{G}_{4} \mathrm{~g}_{41}+\mathrm{G}_{6} \mathrm{~g}_{61}+\mathrm{G}_{9} \mathrm{~g}_{91}=-0.366932 \\
& \mathrm{~L}_{2}=\mathrm{G}_{10} \mathrm{~g}_{102}+\mathrm{G}_{2}+\mathrm{G}_{5} \mathrm{~g}_{52}+\mathrm{G}_{7} \mathrm{~g}_{72}+\mathrm{G}_{8} \mathrm{~g}_{82}+\mathrm{G}_{9} \mathrm{~g}_{92}=-0.380989 \\
& \mathrm{~L}_{3}=\mathrm{G}_{10} \mathrm{~g}_{103 \mathrm{~b}}+\mathrm{G}_{3}+\mathrm{G}_{4} \mathrm{~g}_{43 \mathrm{~b}}+\mathrm{G}_{6} \mathrm{~g}_{63 \mathrm{~b}}+\mathrm{G}_{7} \mathrm{~g}_{73 \mathrm{~b}}+\mathrm{G}_{9} \mathrm{~g}_{93 \mathrm{~b}}=-2.34177 \\
& \mathrm{~L}_{4}=\mathrm{G}_{10} \mathrm{~g}_{104 \mathrm{~b}}+\mathrm{G}_{4}+\mathrm{G}_{6} \mathrm{~g}_{64 \mathrm{~b}}+\mathrm{G}_{7} \mathrm{~g}_{74 \mathrm{~b}}+\mathrm{G}_{8} \mathrm{~g}_{84 \mathrm{~b}}+\mathrm{G}_{9} \mathrm{~g}_{94 \mathrm{~b}}=-0.00702823 \\
& \mathrm{~L}_{5}=\mathrm{G}_{10} \mathrm{~g}_{105 \mathrm{~b}}+\mathrm{G}_{5}+\mathrm{G}_{6} \mathrm{~g}_{65 \mathrm{~b}}+\mathrm{G}_{7} \mathrm{~g}_{75 \mathrm{~b}}+\mathrm{G}_{8} \mathrm{~g}_{85 \mathrm{~b}}+\mathrm{G}_{9} \mathrm{~g}_{95 \mathrm{~b}}=-2.77578
\end{aligned}
$$

The anisotropy tensor $b_{\mathrm{ij}}$ is:

$$
b_{i j}=\left|\begin{array}{ccc}
-0.370838 & -0.154602 & -0.21923 \\
-0.154602 & 0.0155636 & 0.517174 \\
-0.21923 & 0.517174 & 0.355275
\end{array}\right|
$$

2. Using the method one to calculate the anisotropy tensor, to find the matrices $A_{i j}, H_{i j}$, and $J_{i j}$ after that taking the inverse matrix $A_{i j}$, only the first column of its elements are the solutions of the scalar function $\mathrm{L}_{1}, \mathrm{~L}_{2}, \mathrm{~L}_{3}, \mathrm{~L}_{4}$, and $\mathrm{L}_{5}$ as follows:

$$
\begin{aligned}
& \mathrm{L}_{1}=-0.366932 \\
& \mathrm{~L}_{2}=-0.380989 \\
& \mathrm{~L}_{3}=-2.34177 \\
& \mathrm{~L}_{4}=-0.00702823 \\
& \mathrm{~L}_{5}=-2.77578
\end{aligned}
$$

Thus, the solution of the anisotropy tensor $b_{\mathrm{ij}}$ is:

$$
\begin{aligned}
b_{i j} & =L_{1} w_{1}+L_{2} W_{2}+L_{3} W_{3}+L_{4} W_{4}+L_{5} w_{5} \\
b_{i j} & =\left|\begin{array}{ccc}
-0.370838 & -0.154602 & -0.21923 \\
-0.154602 & 0.0155636 & 0.517174 \\
-0.21923 & 0.517174 & 0.355275
\end{array}\right|
\end{aligned}
$$

3. The numerical results for the anisotropy tensor of Gatski and Speziale as

$$
\begin{gathered}
\mathrm{b}_{\mathrm{ij}}=\mathrm{G}_{1} \mathrm{~T}_{1}+\mathrm{G}_{2} \mathrm{~T}_{2}+\mathrm{G}_{3} \mathrm{~T}_{3}+\mathrm{G}_{4} \mathrm{~T}_{4}+\mathrm{G}_{5} \mathrm{~T}_{5}+\mathrm{G}_{6} \mathrm{~T}_{6}+\mathrm{G}_{7} \mathrm{~T}_{7}+\mathrm{G}_{8} \mathrm{~T}_{8}+\mathrm{G}_{9} \mathrm{~T}_{9}+\mathrm{G}_{10} \mathrm{~T}_{10} \\
\mathrm{~b}_{\mathrm{ij}}=\left|\begin{array}{ccc}
-0.370838 & -0.154602 & -0.21923 \\
-0.154602 & 0.0155636 & 0.517174 \\
-0.21923 & 0.517174 & 0.355275
\end{array}\right|
\end{gathered}
$$

4. To compare the present numerical results by using the method one and the method two with the numerical results of Gatski and Speziale, it can be seen that three of them have exactly the same numerical result for the anisotropy tensor.

Case 2: $\mathrm{w}_{3}=\mathbf{0}$ and $\mathrm{w}_{5}=\mathbf{0}$ when $\mathrm{s}_{\mathrm{a}}=\mathrm{s}_{\mathrm{b}}$ or $\mathrm{s}_{\mathrm{b}}=\mathrm{s}_{\mathrm{c}}$ or $\mathrm{s}_{\mathrm{a}}=\mathrm{s}_{\mathrm{c}}$ and $\mathrm{w}_{\mathrm{a}} \neq \mathrm{w}_{\mathrm{b}} \neq \mathrm{w}_{\mathrm{c}}$ where

$$
\mathbf{0}=\{\{0 ., 0 ., 0 .\},\{0 ., 0 ., 0 .,\},\{0 ., 0 ., 0 .\}\}
$$


Enter any random numbers of $\mathrm{S}_{\mathrm{ij}}$ and $\mathrm{W}_{\mathrm{ij}}$ from $[-1,1]$ as follows:

$$
\begin{gathered}
\mathrm{S}_{\mathrm{ij}}=\left|\begin{array}{ccc}
0.5 & 0.0 & 0.0 \\
0.0 & 0.5 & 0.0 \\
0.0 & 0.0 & -1
\end{array}\right| \\
\mathrm{W}_{\mathrm{ij}}=\left|\begin{array}{ccc}
0.0 & -2 / 5 & 3 / 7 \\
2 / 5 & 0.0 & -3 / 4 \\
-3 / 7 & 3 / 4 & 0.0
\end{array}\right|
\end{gathered}
$$

1. Using the method two to calculate the anisotropy tensor, the numerical results as

$$
\begin{gathered}
b_{i j}=\left(G_{1}+G_{10} g_{101}+G_{3} g_{31}+G_{4} g_{41}+G_{6} g_{61}+G_{9} g_{91}\right) w_{1}+ \\
\left(G_{10} g_{102}+G_{2}+G_{5} g_{52}+G_{7} g_{72}+G_{8} g_{82}+G_{9} g_{92}\right) w_{2}+ \\
\left(G_{10} g_{104 b}+G_{4}+G_{6} g_{64 b}+G_{7} g_{74 b}+G_{8} g_{84 b}+G_{9} g_{94 b}\right) w_{4}+ \\
\left(G_{6}+G_{9} g_{96 b}\right) w_{6}+G_{7} w_{7}
\end{gathered}
$$

and the numerical results of $\mathrm{L}_{1}, \mathrm{~L}_{2}, \mathrm{~L}_{4}, \mathrm{~L}_{6}$, and $\mathrm{L}_{7}$ as follows:

$$
\begin{gathered}
\mathrm{L}_{1}=\mathrm{G}_{1}+\mathrm{G}_{10} \mathrm{~g}_{101}+\mathrm{G}_{3} \mathrm{~g}_{31}+\mathrm{G}_{4} \mathrm{~g}_{41}+\mathrm{G}_{6} \mathrm{~g}_{61}+\mathrm{G}_{9} \mathrm{~g}_{91}=-0.407073 \\
\mathrm{~L}_{2}=\mathrm{G}_{10} \mathrm{~g}_{102}+\mathrm{G}_{2}+\mathrm{G}_{5} \mathrm{~g}_{52}+\mathrm{G}_{7} \mathrm{~g}_{72}+\mathrm{G}_{8} \mathrm{~g}_{82}+\mathrm{G}_{9} \mathrm{~g}_{92}=-0.446724 \\
\mathrm{~L}_{4}=\mathrm{G}_{10} \mathrm{~g}_{104 \mathrm{~b}}+\mathrm{G}_{4}+\mathrm{G}_{6} \mathrm{~g}_{64 \mathrm{~b}}+\mathrm{G}_{7} \mathrm{~g}_{74 \mathrm{~b}}+\mathrm{G}_{8} \mathrm{~g}_{84 \mathrm{~b}}+\mathrm{G}_{9} \mathrm{~g}_{94 \mathrm{~b}}=-0.0144359 \\
\mathrm{~L}_{6}=\mathrm{G}_{6}+\mathrm{G}_{9} \mathrm{~g}_{96 \mathrm{~b}}=-0.703069 \\
\mathrm{~L}_{7}=\mathrm{G}_{7}=0.351534
\end{gathered}
$$

The anisotropy tensor $b_{\mathrm{ij}}$ is:

$$
\text { bij }=\left|\begin{array}{ccc}
-0.434091 & -0.0812456 & -0.121203 \\
-0.0812456 & 0.0270173 & 0.597408 \\
-0.121203 & 0.597408 & 0.407073
\end{array}\right|
$$

2. Using the method one to calculate the anisotropy tensor, to find the matrices $A_{i j}, H_{i j}$, and $J_{i j}$ after that taking the inverse matrix $\mathrm{A}_{\mathrm{ij}}$, only the first column of its elements are the solutions of the scalar function $\mathrm{L}_{1}, \mathrm{~L}_{2}, \mathrm{~L}_{4}, \mathrm{~L}_{6}$ and $\mathrm{L}_{7}$ as follows:

$$
\begin{aligned}
& \mathrm{L}_{1}=-0.407073 \\
& \mathrm{~L}_{2}=-0.446724 \\
& \mathrm{~L}_{4}=-0.0144359 \\
& \mathrm{~L}_{6}=-0.703069 \\
& \mathrm{~L}_{7}=0.351534
\end{aligned}
$$

Thus, the solution of the anisotropy tensor $b_{i j}$ is:

$$
\begin{gathered}
\mathrm{b}_{\mathrm{ij}}=\mathrm{L}_{1} \mathrm{w}_{1}+\mathrm{L}_{2} \mathrm{~W}_{2}+\mathrm{L}_{4} \mathrm{w}_{4}+\mathrm{L}_{6} \mathrm{~W}_{6}+\mathrm{L}_{7} \mathrm{~W}_{7} \\
\mathrm{~b}_{\mathrm{ij}}=\left|\begin{array}{ccc}
-0.434091 & -0.0812456 & -0.121203 \\
-0.0812456 & 0.0270173 & 0.597408 \\
-0.121203 & 0.597408 & 0.407073
\end{array}\right|
\end{gathered}
$$

3. The numerical results for the anisotropy tensor of Gatski and Speziale as

$$
\begin{gathered}
\mathrm{b}_{\mathrm{ij}}=\mathrm{G}_{1} \mathrm{~T}_{1}+\mathrm{G}_{2} \mathrm{~T}_{2}+\mathrm{G}_{3} \mathrm{~T}_{3}+\mathrm{G}_{4} \mathrm{~T}_{4}+\mathrm{G}_{5} \mathrm{~T}_{5}+\mathrm{G}_{6} \mathrm{~T}_{6}+\mathrm{G}_{7} \mathrm{~T}_{7}+\mathrm{G}_{8} \mathrm{~T}_{8}+\mathrm{G}_{9} \mathrm{~T}_{9}+\mathrm{G}_{10} \mathrm{~T}_{10} \\
\mathrm{~b}_{\mathrm{ij}}=\left|\begin{array}{ccc}
-0.434091 & -0.0812456 & -0.121203 \\
-0.0812456 & 0.0270173 & 0.597408 \\
-0.121203 & 0.597408 & 0.407073
\end{array}\right|
\end{gathered}
$$

4. To compare the present numerical results by using the method one and the method two with the numerical results of Gatski and Speziale, it can be seen that three of them have exactly the same numerical result for the anisotropy tensor. 


\section{Discussion of the Present Research}

The present numerical results are compared with the experimental data of Tavoularis \& Corrsin (1981) and the other results. From these results, it is clear that:

1). The numerical results of present research are far better than the other results.

2). The numerical results of present research are close to the experiment data, with an error of approximately 2 to 3 percent.

3). The results of FLT model is better than the other methods and very close with the experimental data and the present research.

4). The results of RK model is also better and very close with the results of FLT model, the present research, and the experimental data.

5). It is clear from these results that the present research has excellent results and very close to the experimental data.

The comparatively good predictions obtained from the second order closures are largely due to the fact that the production and Coriolis terms in equation (3.44), which make a major contribution in determining the structure of rotating shear flow, are accounted for exactly. However, like the other existing second order closures, it does not yield accurate results when there is both shear and rotation, the development of significantly improved models based on invariance arguments and dynamical systems approach will be subject in future research.

At the present time there are many variations of second order closure turbulence models available. However there is no unique turbulence model exists which can predict satisfactorily all turbulence flows. Each turbulence model applies successfully to some turbulent flows, but predicts unsatisfactorily to others.

\section{Conclusion}

The modern development of turbulence modeling began in the 1940s and 1950s, and the applications of second order turbulence modeling began in the 1960s when computer became available to handle the computations that required using the advanced turbulence models (Taulbee, 1992; Johansson \& Wallin, 1996). In the 1970s, the applications of turbulence modeling became very popular; however, most of the applications and calculations were two dimensional (Abid \& Speziale, 1992). In the 1980s, the computations and applications have been extended to three dimensional problems and the use of more turbulence models, many of them were based on the Reynolds stress models (Ching-Jen \& Shenq-yuh, 1997). Although there are other approaches of turbulence modeling, but the most popular trend at the present time and in the future is the Reynolds stress model with its applications and computations (Wilcox, 1993). Each application of turbulence models is successfully to some turbulent flows, but predicts unsatisfactorily to others.

The explicit algebraic Reynolds stress models of the present research for three dimensional turbulent flows in non inertial frames have been obtained from the second order closure models that involve seven tensor polynomial groups with six independent invariants. The anisotropy tensor of the present research looks complicated but easy to use and apply because it requires less computational times.

This present research only used 49 terms to calculate for the anisotropy tensor while Gatski and Speziale model used 100 terms to calculate the anisotropy tensor. The present results of the explicit algebraic Reynolds stress models can apply and predict the turbulent flows by leading to second order closures in the applications and calculations of turbulent flows.

\section{References}

Abid, R., \& Speziale, C. G. (1992). Predicting equilibrium states with Reynolds stress clousres in channel flow and homogenous shear flow. ICASE Report 92-28, Univ. Space research Assoc., Hampton, VA.

Ching-Jen, C., \& Shenq-yuh, J. (1997). Fundamentals of turbulence modeling. Combustion: An international series.

Daly, B. J., \& Harlow, F. H. (1970). Transport equations in turbulence. Physics of Fluids, 13, 2634-2649. http://dx.doi.org/10.1063/1.1692845

Gatski, T. B., \& Speziale, C. G. (1993). On explicit algebraic stress models for complex turbulent flows. J. Fluid Mech., 254.

Johansson, A. V., \& Wallin, S. (1996). A new explicit algebraic Reynolds stress model. Proc. Sixth European Turbulence Conference, Lausanne, July 1996, Ed. P. Monkewitz. 
Launder, B. E., Reece, G., \& Rodi, W. (1975). Progress in the development of a Reynolds stress turbulence closures. J. Fluid Mech., 41.

Lumley, J. L. (1970). Toward a turbulent constitutive equation. J. Fluid Mech., 41, 413-434. http://dx.doi.org/10.1017/S0022112070000678

Lumley, J. L. (1983). Turbulence modeling. ASME J. App. Mech., 50, pp. 1097-1103.

Pope, S. B. (1975). A more general effective viscosity hypothesis. J. Fluid Mech., 72, 331-340. http://dx.doi.org/10.1017/S0022112075003382

Rodi, W. (1996). A new algebraic relation for calculating the Reynolds stresses Z. angew. Math. Mech., 56, 219-221.

Rotta, J. (1951). Statische theorie nichthomogener turbulenz. Zeitschrift für physik a hadrons and nuclei, 131(1), 51-77.

Spencer, A. J. M. (1971). Theory of invariants. In continuum Physics (ed. A. C. Eringen), 1, 240-352. Academic. A. J. M.

Spencer, \& Rivin, R. S. (1958). The theory of matrix polynomials and its application to the mechanics of is otropic continua. Arch. Rational Mech. Anal., 2, 308-336.

Taulbee, D. B. (1992). An improved algebraic Reynolds stress model and corresponding nonlinear stress model. Phys. Fluids A, 4, 2555-2561. http://dx.doi.org/10.1063/1.858442

Wilcox, D. C. (1993). Turbulence modeling for CFD. DCW Industries, Inc. La Canada, CA. 